\title{
Bioelectric Field Imaging
}

National Cancer Institute

\section{Source}

National Cancer Institute. Bioelectric Field Imaging. NCI Thesaurus. Code C116485.

Noninvasive measurement of the bioelectric fields across the epidermis. 